\title{
Modular symmetry and non-Abelian discrete flavor symmetries in string compactification
}

\author{
Tatsuo Kobayashi, Satoshi Nagamoto, Shintaro Takada, Shio Tamba, and Takuya H. Tatsuishi \\ Department of Physics, Hokkaido University, Sapporo 060-0810, Japan
}

(Received 25 April 2018; published 5 June 2018)

\begin{abstract}
We study the modular symmetry in magnetized D-brane models on $T^{2}$. Non-Abelian flavor symmetry $D_{4}$ in the model with magnetic flux $M=2$ (in a certain unit) is a subgroup of the modular symmetry. We also study the modular symmetry in heterotic orbifold models. The $T^{2} / Z_{4}$ orbifold model has the same modular symmetry as the magnetized brane model with $M=2$, and its flavor symmetry $D_{4}$ is a subgroup of the modular symmetry.
\end{abstract}

DOI: $10.1103 /$ PhysRevD.97.116002

\section{INTRODUCTION}

Non-Abelian discrete flavor symmetries play an important role in particle physics. In particular, many models with various finite groups have been studied in order to explain quark and lepton masses and their mixing angles. (See for review [1-3].) Those symmetries may be useful for dark matter physics and multi-Higgs models.

Superstring theory is a promising candidate for unified theory including gravity. It has been shown that some nonAbelian discrete flavor symmetries appear in superstring theory with certain compactifications. Heterotic string theory on toroidal $Z_{N}$ orbifolds can realize non-Abelian flavor symmetries, e.g., $D_{4}$, and $\Delta(54)$ [4]. (See also $\left.[5,6].\right)^{1}$ Furthermore, magnetized D-brane models within the framework of type II superstring theory can lead to similar flavor symmetries [8-12]. Intersecting D-brane models are T-dual to magnetized D-brane models. Then, one can realize the same aspects in intersecting D-brane models as in the magnetized ones. ${ }^{2}$

On the other hand, superstring theories on tori and orbifolds have modular symmetry. Recently, the behavior of zero modes under modular transformation was studied in magnetized D-brane models in Ref. [14]. (See also [15].) Also, the behavior of twisted sectors under modular transformation was already studied in Refs. [16-18]. These modular transformations also act nontrivially on flavors and transform mutually flavors from each other.

\footnotetext{
${ }^{1}$ In Ref. [7], a relation between gauge symmetries and nonAbelian flavor symmetries is discussed at the enhancement point.

${ }^{2}$ See also [13].

Published by the American Physical Society under the terms of the Creative Commons Attribution 4.0 International license. Further distribution of this work must maintain attribution to the author(s) and the published article's title, journal citation, and DOI. Funded by SCOAP ${ }^{3}$.
}

The remarkable difference is that modular transformation also acts on Yukawa couplings as well as higher order couplings, while those couplings are trivial singlets under the usual non-Abelian symmetries.

The purpose of this paper is to study more how modular transformation is represented by zero modes in magnetized D-brane models, and to discuss relations between modular transformation and non-Abelian flavor symmetries in magnetized D-brane models. Intersecting D-brane models have the same aspects as magnetized D-brane models, because they are T-dual to each other. Furthermore, intersecting D-brane models in type II superstring theory and heterotic string theory have similarities, e.g., in twodimensional conformal field theory. Thus, here we study modular symmetry and non-Abelian discrete flavor symmetries in heterotic orbifold models, too.

This paper is organized as follows. In Sec. II, we study the modular symmetries in magnetized D-brane models and the relation to the $D_{4}$ flavor symmetry. In Sec. III, we study the modular symmetries in heterotic orbifold models. Section IV is the conclusion and discussions. We give brief reviews on non-Abelian discrete flavor symmetries in magnetized D-brane models and heterotic orbifold models in Appendixes A and B, respectively.

\section{MODULAR TRANSFORMATION IN MAGNETIZED D-BRANE MODELS}

In this section, we study the modular transformation of zero-mode wave functions in magnetized D-brane models.

\section{A. Zero-mode wave function}

Here, we give a brief review on zero-mode wave functions on torus with magnetic flux [15]. For simplicity, we concentrate on $T^{2}$ with $U(1)$ magnetic flux. The complex coordinate on $T^{2}$ is denoted by $z=x^{1}+\tau x^{2}$, where $\tau$ is 
the complex modular parameter, and $x^{1}$ and $x^{2}$ are real coordinates. The metric on $T^{2}$ is given by

$$
g_{\alpha \beta}=\left(\begin{array}{ll}
g_{z z} & g_{z \bar{z}} \\
g_{\bar{z} z} & g_{\bar{z} \bar{z}}
\end{array}\right)=(2 \pi R)^{2}\left(\begin{array}{cc}
0 & \frac{1}{2} \\
\frac{1}{2} & 0
\end{array}\right) \text {. }
$$

We identify $z \sim z+1$ and $z \sim z+\tau$ on $T^{2}$.

On $T^{2}$, we introduce the $U(1)$ magnetic flux $F$,

$$
F=i \frac{\pi M}{\operatorname{Im} \tau}(d z \wedge d \bar{z}),
$$

which corresponds to the vector potential,

$$
A(z)=\frac{\pi M}{\operatorname{Im} \tau} \operatorname{Im}(\bar{z} d z) .
$$

Here we concentrate on vanishing Wilson lines.

In the above background, we consider the zero-mode equation for the spinor field with the $U(1)$ charge $q=1$,

$$
i \not \Psi=0 .
$$

The spinor field on $T^{2}$ has two components,

$$
\Psi(z, \bar{z})=\left(\begin{array}{l}
\psi_{+} \\
\psi_{-}
\end{array}\right) .
$$

The magnetic flux should be quantized such that $M$ is an integer. Either $\psi_{+}$or $\psi_{-}$has zero modes exclusively for $M \neq 0$. For example, we set $M$ to be positive. Then, $\psi_{+}$ has $M$ zero modes, while $\psi_{-}$has no zero mode. Hence, we can realize a chiral theory. Their zero-mode profiles are given by

$$
\psi^{j, M}(z)=\mathcal{N} e^{i \pi M z \frac{\mathrm{Im} z}{\mathrm{~m} \tau}} \cdot \vartheta\left[\begin{array}{c}
\frac{j}{M} \\
0
\end{array}\right](M z, M \tau),
$$

with $j=0,1, \ldots,(M-1)$, where $\vartheta$ denotes the Jacobi theta function,

$$
\vartheta\left[\begin{array}{l}
a \\
b
\end{array}\right](\nu, \tau)=\sum_{l \in \mathbf{Z}} e^{\pi i(a+l)^{2} \tau} e^{2 \pi i(a+l)(\nu+b)} .
$$

Here, $\mathcal{N}$ denotes the normalization factor given by

$$
\mathcal{N}=\left(\frac{2 \operatorname{Im} \tau M}{\mathcal{A}^{2}}\right)^{1 / 4}
$$

with $\mathcal{A}=4 \pi^{2} R^{2} \operatorname{Im} \tau$.

The ground states of scalar fields also have the same profiles as $\psi^{j, M}$. Thus, the Yukawa coupling including one scalar and two spinor fields can be computed by using these zero-mode wave functions. Zero mode wave functions satisfy the following relation:

$$
\begin{aligned}
\psi^{i, M} \psi^{j, M}= & \mathcal{A}^{-1 / 2}(2 \operatorname{Im} \tau)^{1 / 4}\left(\frac{M N}{M+N}\right)^{1 / 4} \\
& \times \sum_{m} \psi^{i+j+M m, M+N} \\
& \cdot \vartheta\left[\begin{array}{c}
\frac{N i-M j+M N m}{M N(M+N)} \\
0
\end{array}\right](0, M N(M+N) \tau) .
\end{aligned}
$$

By use of this relation, their Yukawa couplings are given by the wave function overlap integral,

$$
\begin{aligned}
Y_{i j k}= & y \int d^{2} z \psi^{i, M} \psi^{j, N}\left(\psi^{k, M^{\prime}}\right)^{*} \\
= & y\left(\frac{2 \operatorname{Im} \tau}{\mathcal{A}^{2}}\right)^{1 / 4} \sum_{m \in Z_{M^{\prime}}} \delta_{k, i+j+M m} \\
& \cdot \vartheta\left[\begin{array}{c}
\frac{N i-M j+M N m}{M N M^{\prime}} \\
0
\end{array}\right]\left(0, M N M^{\prime} \tau\right),
\end{aligned}
$$

where $y$ is constant. This Yukawa coupling vanishes for $M^{\prime} \neq M+N$. Similarly, we can compute higher order couplings using the relation (9) [19]. In the above equation, the Kronecker delta $\delta_{k, i+j+M m}$ implies the coupling selection rule. For $g=\operatorname{gcd}\left(M, N, M^{\prime}\right)$, nonvanishing Yukawa couplings appear only if

$$
i+j=k \quad(\bmod g) .
$$

Hence, we can define $Z_{g}$ charges in these couplings [8].

\section{B. Modular transformation}

Here, we study modular transformation. First, we give a brief review on results of modular transformation [14]. (See also [15].) Then, we will study in more detail.

The $T^{2}$ is constructed by $\mathbb{R}^{2} / \Lambda$, and the lattice $\Lambda$ is spanned by the vectors $\left(\alpha_{1}, \alpha_{2}\right)$, where $\alpha_{1}=2 \pi R$ and $\alpha_{2}=2 \pi R \tau$. However, the same lattice can be described by another basis,

$$
\left(\begin{array}{c}
\alpha_{2}^{\prime} \\
\alpha_{1}^{\prime}
\end{array}\right)=\left(\begin{array}{ll}
a & b \\
c & d
\end{array}\right)\left(\begin{array}{c}
\alpha_{2} \\
\alpha_{1}
\end{array}\right),
$$

where $a, b, c, d$ are integers satisfying $a d-b c=1$. That is the $S L(2, Z)$ transformation.

The modular parameter $\tau=\alpha_{2} / \alpha_{1}$ transforms as

$$
\tau \rightarrow \frac{a \tau+b}{c \tau+d},
$$

under (12). This transformation includes two important generators, $S$ and $T$, 


$$
\begin{gathered}
S: \tau \rightarrow-\frac{1}{\tau}, \\
T: \tau \rightarrow \tau+1 .
\end{gathered}
$$

They satisfy

$$
S^{2}=\mathbb{I}, \quad(S T)^{3}=\mathbb{I} .
$$

On top of that, if we impose the algebraic relation,

$$
T^{N}=\mathbb{I},
$$

that corresponds to the congruence subgroup of modular group, $\Gamma(N)$. For example, it is found that $\Gamma(2) \simeq S_{3}$, $\Gamma(3) \simeq A_{4}, \Gamma(4) \simeq S_{4}$, and $\Gamma(5) \simeq A_{5}$. Since group $A_{4}$ has the symmetry of the tetrahedron, it is often called the tetrahedral group $T=A_{4}$. Also, it may be useful to mention about $\Delta\left(3 N^{2}\right) \simeq\left(Z_{N} \times Z_{N}\right) \rtimes Z_{3}$ and $\Delta\left(6 N^{2}\right) \simeq$ $\left(Z_{N} \times Z_{N}\right) \rtimes S_{3}$. We find that $S_{3} \simeq \Delta(6), A_{4} \simeq \Delta(12)$, and $S_{4} \simeq \Delta(24)$.

Following [14], we restrict ourselves to even magnetic fluxes $M(M>0)$. Under $S$, the zero-mode wave functions transform as $[14,15]$

$$
\psi^{j, M} \rightarrow \frac{1}{\sqrt{M}} \sum_{k} e^{2 \pi i j k / M} \psi^{k, M} .
$$

On the other hand, the zero-mode wave functions transform as [14]

$$
\psi^{j, M} \rightarrow e^{\pi i j^{2} / M} \psi^{j, M},
$$

under $T$. Generically, the $T$ transformation satisfies

$$
T^{2 M}=\mathbb{I},
$$

on the zero modes, $\psi^{j, M}$. Furthermore, in Ref. [14] it is shown that

$$
(S T)^{3}=e^{\pi i / 4},
$$

on the zero modes, $\psi^{j, M}$.

In what follows, we study more concretely.

\section{Magnetic flux $M=2$}

Let us study the case with the magnetic flux $M=2$ concretely. There are two zero modes, $\psi^{0,2}$ and $\psi^{1,2}$. The $S$ transformation acts on these zero modes as

$$
\left(\begin{array}{l}
\psi^{0,2} \\
\psi^{1,2}
\end{array}\right) \rightarrow S_{(2)}\left(\begin{array}{l}
\psi^{0,2} \\
\psi^{1,2}
\end{array}\right), \quad S_{(2)}=\frac{1}{\sqrt{2}}\left(\begin{array}{cc}
1 & 1 \\
1 & -1
\end{array}\right) .
$$

The $T$ transformation acts as

$$
\left(\begin{array}{l}
\psi^{0,2} \\
\psi^{1,2}
\end{array}\right) \rightarrow T_{(2)}\left(\begin{array}{l}
\psi^{0,2} \\
\psi^{1,2}
\end{array}\right), \quad T_{(2)}=\left(\begin{array}{ll}
1 & 0 \\
0 & i
\end{array}\right) .
$$

They satisfy the following algebraic relations:

$$
S_{(2)}^{2}=\mathbb{I}, \quad T_{(2)}^{4}=\mathbb{I}, \quad\left(S_{(2)} T_{(2)}\right)^{3}=e^{\pi i / 4} \mathbb{I} .
$$

They construct a closed algebra with the order 192, which we denote here by $G_{(2)}$. By such an algebra, the modular transformation is represented by two zero modes, $\psi^{0,2}$ and $\psi^{1,2}$. We find that $\left(S_{(2)} T_{(2)}\right)^{3}$ is a center. Indeed, there are eight center elements and their group is $Z_{8}$. Other diagonal elements correspond to $Z_{4}$, which is generated by $T_{(2)}$. Here, we denote

$$
a=\left(S_{(2)} T_{(2)}\right)^{3}, \quad a^{\prime}=T_{(2)} .
$$

The diagonal elements are represented by $a^{m} a^{\prime n}$, i.e., $Z_{8} \times Z_{4}$.

Here, we examine the right coset $H g$ for $g \in G_{(2)}$, where $H$ is the above $Z_{8} \times Z_{4}$, i.e., $H=\left\{a^{m} a^{\prime n}\right\}$. There would be $6(=192 /(8 \times 4))$ cosets. Indeed, we obtain the following six cosets:

$$
H, \quad H S_{(2)}, \quad H S_{(2)} T_{(2)}^{k}, \quad H S_{(2)} T_{(2)}^{2} S_{(2)},
$$

with $k=1,2,3$. By simple computations, we find $H S_{(2)} T_{(2)}^{k}$ $S_{(2)} \sim H S_{(2)} T_{(2)}^{4-k} S_{(2)}$ and $H S_{(2)} T_{(2)}^{2} S_{(2)} T \sim H S_{(2)} T_{(2)}^{2} S_{(2)}$.

Furthermore, we would make a (non-Abelian) subgroup with the order 6 by choosing properly six elements such that we pick one element up from each coset and their algebra is closed. The non-Abelian group with the order 6 is unique, i.e., $S_{3}$. For example, we may be able to obtain the $Z_{3}$ generator from $H S_{(2)} T_{(2)}$ because $\left(S_{(2)} T_{(2)}\right)^{3} \in H$. That is, we define

$$
b=a^{m} a^{\prime n} S_{(2)} T_{(2)}
$$

Then, we require $b^{3}=\mathbb{I}$. There are three solutions, $(m, n)=(3,2),(5,0) \bmod (8,4)$. Similarly, we can obtain the $Z_{2}$ generator, e.g., from $H S_{(2)} T_{(2)}^{2} S_{(2)}$ because $\left(S_{(2)} T_{(2)}^{2} S_{(2)}\right)^{2} \in H$. Then, we define

$$
c=a^{m^{\prime}} a^{\prime n^{\prime}} S_{(2)} T_{(2)}^{2} S_{(2)} .
$$

We find $c^{2}=\mathbb{I}$ when $n^{\prime}=-m^{\prime} \bmod 4$. On top of that, we require $(b c)^{2}=\mathbb{I}$, and that leads to the conditions $n=$ $-m^{\prime}-1 \bmod 4$ and $m=m^{\prime}+2 \bmod 8$. As a result, there are six solutions, $\left(m, n, m^{\prime}\right)=(3,2,1),(3,2,5),(5,0,3)$, $(5,0,7)$ with $n^{\prime}=-m^{\prime} \bmod 4$. 
For example, for $\left(m, n, m^{\prime}\right)=(3,2,5)$ we write

$$
b=\frac{1}{\sqrt{2}}\left(\begin{array}{cc}
\rho^{3} & \rho^{-3} \\
\rho^{-1} & \rho^{-3}
\end{array}\right), \quad c=\left(\begin{array}{cc}
0 & \rho^{-3} \\
\rho^{3} & 0
\end{array}\right) .
$$

The six elements of the subgroup are written explicitly,

$$
\begin{aligned}
& \left(\begin{array}{ll}
1 & 0 \\
0 & 1
\end{array}\right), \quad \frac{1}{\sqrt{2}}\left(\begin{array}{cc}
1 & 1 \\
1 & -1
\end{array}\right), \quad\left(\begin{array}{cc}
0 & \rho^{-3} \\
\rho^{3} & 0
\end{array}\right), \quad \frac{1}{\sqrt{2}}\left(\begin{array}{ll}
-1 & i \\
-i & 1
\end{array}\right), \\
& \frac{1}{\sqrt{2}}\left(\begin{array}{cc}
\rho^{3} & \rho^{-3} \\
\rho^{-1} & \rho^{-3}
\end{array}\right), \quad \frac{1}{\sqrt{2}}\left(\begin{array}{cc}
\rho^{-3} & \rho \\
\rho^{3} & \rho^{3}
\end{array}\right),
\end{aligned}
$$

where $\rho=e^{2 \pi i / 8}$. They correspond to $S_{3} \simeq \Gamma(2) \simeq \Delta(6)$ because they satisfy the following algebraic relations:

$$
c^{2}=b^{3}=(b c)^{2}=\mathbb{I} .
$$

Moreover, they satisfy the following algebraic relation with $Z_{8} \times Z_{4}$ :

$$
\begin{aligned}
b^{-1} a b^{1} & =a, \quad c a c=a, \quad b^{-1} a^{\prime} b=a, \\
c a^{\prime} c^{-1} & =a^{2} a^{\prime 3} .
\end{aligned}
$$

Thus, the algebra of $G_{(2)}$ is isomorphic to $\left(Z_{8} \times Z_{4}\right) \rtimes S_{3}$.

We have started by choosing $H S_{(2)} T_{(2)}^{2} S_{(2)}$ for a candidate of the $Z_{2}$ generator. We can obtain the same results by starting with $H S_{(2)}$ for a candidate of the $Z_{2}$ generator.

\section{Magnetic flux $M=4$}

Similarly, we study the case with the magnetic flux $M=4$. There are four zero modes, $\psi^{i, M}$ with $i=0,1,2,3$. The $S$ and $T$ transformations are represented by $\psi^{i, M}$,

$$
\begin{aligned}
S_{(4)} & =\frac{1}{2}\left(\begin{array}{cccc}
1 & 1 & 1 & 1 \\
1 & i & -1 & -i \\
1 & -1 & 1 & -1 \\
1 & -i & -1 & i
\end{array}\right), \\
T_{(4)} & =\left(\begin{array}{cccc}
1 & & & \\
& e^{\pi i / 4} & & \\
& & -1 & \\
& & & e^{\pi i / 4}
\end{array}\right) .
\end{aligned}
$$

This is a reducible representation. In order to obtain irreducible representations, we use the flowing basis,

$$
\begin{aligned}
\left(\begin{array}{c}
\psi^{0.4} \\
\psi_{+}^{1,4} \\
\psi^{2,4}
\end{array}\right) & =\left(\begin{array}{c}
\psi^{0.4} \\
\frac{1}{\sqrt{2}}\left(\psi^{1,4}+\psi^{3,4}\right) \\
\psi^{2,4}
\end{array}\right), \\
\psi_{-}^{1,4} & =\frac{1}{\sqrt{2}}\left(\psi^{1,4}-\psi^{3,4}\right) .
\end{aligned}
$$

This is nothing but zero modes on the $T^{2} / Z_{2}$ orbifold [20]. The former corresponds to $Z_{2}$ even states, while the latter corresponds to the $Z_{2}$ odd state. Note that $(S T)^{3}$ transforms the lattice basis $\left(\alpha_{1}, \alpha_{2}\right) \rightarrow\left(-\alpha_{1},-\alpha_{2}\right)$. Thus, it is reasonable that the zero modes on the $T^{2} / Z_{2}$ orbifold correspond to the irreducible representations.

The $S$ and $T$ representations by the $Z_{2}$ odd zero mode are quite simple, and these are represented by

$$
S_{(4-)}=i, \quad T_{(4)-}=e^{\pi i / 4} .
$$

Their closed algebra is $Z_{8}$.

On the other hand, the $S$ and $T$ transformations are represented by the $Z_{2}$ even zero- modes,

$$
\begin{aligned}
& S_{(4)+}=\frac{1}{2}\left(\begin{array}{ccc}
1 & \sqrt{2} & 1 \\
\sqrt{2} & 0 & -\sqrt{2} \\
1 & -\sqrt{2} & 1
\end{array}\right), \\
& T_{(4)+}=\left(\begin{array}{ccc}
1 & & \\
& e^{\pi i / 4} & \\
& & -1
\end{array}\right) .
\end{aligned}
$$

They satisfy the following algebraic relation:

$$
\left(S_{(4)+}\right)^{2}=\mathbb{I}, \quad\left(T_{(4)+}\right)^{8}=\mathbb{I}, \quad\left(S_{(4)+} T_{(4)+}\right)^{3}=e^{\pi i / 4} \mathbb{I} .
$$

We denote the closed algebra of $S_{(4)+}$ and $T_{(4)+}$ by $G_{(4)+}$. Its order is equal to 768 , and it includes the center element $\left(S_{(4)+} T_{(4)+}\right)^{3}$, i.e., $Z_{8}$. Other diagonal elements correspond to $Z_{8}$, which is generated by $T_{(4)+}$. Again, we denote $a=$ $\left(S_{(4)+} T_{(4)+}\right)^{3}$ and $a^{\prime}=T_{(4)+}$, and the diagonal elements are written by $a^{m} a^{\prime n}$, i.e., $Z_{8} \times Z_{8}$.

Similar to the case with $M=2$, we examine the coset structure, $\mathrm{Hg}$. Indeed, there are the following 12 cosets:

$$
H, \quad H S_{(4)+}, \quad H S_{(4)+}^{k}, \quad H S_{(4)+} T_{(4)+}^{\ell} S_{(4)+},
$$

where $k=1, \ldots, 7$ and $\ell=2,4,6$. By simple computation, we find that

$$
\begin{aligned}
& H S_{(4)+} T_{(4)+}^{k} S_{(4)+} \sim H S_{(4)+} T_{(4)+}^{8-k}, \\
& H S_{(4)+} T_{(4)+}^{\ell} S_{(4)+} T \sim H S_{(4)+} T_{(4)+}^{8-\ell} S_{(4)+}
\end{aligned}
$$

for $k=$ odd and $\ell=$ even.

We make a subgroup with the order 12 by choosing properly 12 elements such that we pick one element up from each coset and their algebra is closed. The nonAbelian group with the order 12 are $D_{6}, Q_{6}$, and $A_{4}$. Among them, $A_{4}$ would be a good candidate. Indeed, we can obtain the $Z_{3}$ generator from $H S_{(4)+} T_{(4)+}$, gain. That is, we define 


$$
t=a^{m} a^{\prime n} S_{(4)+} T_{(4)+} \cdot
$$

The solutions for $t^{3}=\mathbb{I}$ are obtained by $(m, n)=(1,4)$, $(3,6),(5,0)$, and $(7,2)$. We also define

$$
s=a^{m^{\prime}} a^{\prime n^{\prime}} S_{(4)+} T_{(4)+}^{4} S_{(4)+} .
$$

The solutions for $s^{2}=\mathbb{I}$ are obtained by $\left(m^{\prime}, n^{\prime}\right)=(0,0)$, $(0,4),(4,0)$, and $(4,4)$. These two generators satisfy $(s t)^{3}=\mathbb{I}$ if $\left(m^{\prime}, n^{\prime}\right)=(0,4)$, and $(4,0)$, i.e.,

$$
s=\left(\begin{array}{ccc}
0 & 0 & \pm 1 \\
0 & -1 & 0 \\
\pm 1 & 0 & 0
\end{array}\right)
$$

As a result, they satisfy

$$
s^{2}=t^{3}=(s t)^{3}=\mathbb{I} .
$$

That is the $A_{4}$ algebra.

\section{Large magnetic flux $M$}

For larger magnetic fluxes, $S$ and $T$ transformations are represented by zero modes $\psi^{j, M}$, but those are reducible representations. The irreducible representations are obtained in the $T^{2} / Z_{2}$ orbifold basis,

$$
\psi_{ \pm}^{j, M}=\frac{1}{\sqrt{2}}\left(\psi^{j, M} \pm \psi^{M-j, j}\right)
$$

The representations of $T_{(M)}$ are simply obtained by

$$
\begin{aligned}
& T_{(M)+}\left(\begin{array}{c}
\psi^{0, M} \\
\psi_{+}^{1, M} \\
\vdots \\
\psi_{+}^{j, M} \\
\vdots \\
\psi^{M / 2, M}
\end{array}\right)=\left(\begin{array}{cccccc}
1 & & & & & \\
& e^{\pi i / M} & & & & \\
& & \ddots & & & \\
& & & e^{\pi i j^{2} / M} & & \\
& & & \ddots & \\
& & & & & e^{\pi i M / 4}
\end{array}\right) \\
& \times\left(\begin{array}{c}
\psi^{0, M} \\
\psi_{+}^{1, M} \\
\vdots \\
\psi_{+}^{j, M} \\
\vdots \\
\psi^{M / 2, M}
\end{array}\right)
\end{aligned}
$$

and

$$
T_{(M)-}\left(\begin{array}{c}
\psi_{-}^{1, M} \\
\vdots \\
\psi_{-}^{j, M} \\
\vdots \\
\psi_{-}^{M / 2-1, M}
\end{array}\right)=\left(\begin{array}{ccccc}
e^{\pi i / M} & & & & \\
& \ddots & & & \\
& & e^{\pi i j^{2} / M} & & \\
& & & \ddots & \\
& & & & e^{\pi(M / 2-1)^{2} / M}
\end{array}\right)\left(\begin{array}{c}
\psi_{-}^{1, M} \\
\vdots \\
\psi^{j, M} \\
\vdots \\
\psi_{-}^{M / 2-1, M}
\end{array}\right) .
$$

Thus, the $S$ transformation is represented on the $T^{2} / Z_{2}$ orbifold basis by

$S_{(M) \pm} \psi_{ \pm}^{j, M}=\frac{1}{\sqrt{M}} \sum_{k \leq M / 2}\left(e^{2 \pi j k / M} \pm e^{2 \pi i(M-j) k / M}\right) \psi_{ \pm}^{k, M}$.

This representation is also written by

$$
\begin{aligned}
& S_{(M) \pm} \psi_{ \pm}^{j, M} \\
& \quad=\frac{1}{\sqrt{2 M}} \sum_{k}\left(e^{2 \pi(M-j)(M-k) / M} \pm e^{2 \pi i j(M-k) / M, M}\right) \psi^{M-k, M} .
\end{aligned}
$$

These are written by

$$
\begin{aligned}
& S_{(M)+} \psi_{+}^{j, M}=\frac{2}{\sqrt{M}} \sum_{k \leq M / 2} \cos (2 \pi j k / M) \psi_{+}^{j, M}, \\
& S_{(M)-} \psi_{-}^{j, M}=\frac{2 i}{\sqrt{M}} \sum_{k \leq M / 2} \sin (2 \pi j k / M) \psi_{-}^{j, M} .
\end{aligned}
$$

For example, for $M=6, S$ and $T$ are represented by $Z_{2}$ even zero modes, 


$$
S_{(6)+}\left(\begin{array}{c}
\psi^{0,6} \\
\psi_{+}^{1,6} \\
\psi_{+}^{2,6} \\
\psi^{3,6}
\end{array}\right)=\frac{1}{\sqrt{6}}\left(\begin{array}{cccc}
1 & \sqrt{2} & \sqrt{2} & 1 \\
\sqrt{2} & 1 & -1 & -\sqrt{2} \\
\sqrt{2} & -1 & -1 & \sqrt{2} \\
1 & -\sqrt{2} & \sqrt{2} & -1
\end{array}\right)\left(\begin{array}{c}
\psi^{0,6} \\
\psi_{+}^{1,6} \\
\psi_{+}^{2,6} \\
\psi^{3,6}
\end{array}\right),
$$

$$
T_{(6)+}\left(\begin{array}{c}
\psi^{0,6} \\
\psi_{+}^{1,6} \\
\psi_{+}^{2,6} \\
\psi^{3,6}
\end{array}\right)=\left(\begin{array}{cccc}
1 & & & \\
& e^{\pi i / 6} & & \\
& & e^{2 \pi i / 3} & \\
& & & e^{3 \pi i / 2}
\end{array}\right)\left(\begin{array}{c}
\psi^{0,6} \\
\psi_{+}^{1,6} \\
\psi_{+}^{2,6} \\
\psi^{3,6}
\end{array}\right),
$$

while $S$ and $T$ are represented by $Z_{2}$ odd zero mode,

$$
\begin{aligned}
S_{(6)-}\left(\begin{array}{c}
\psi_{-}^{1,6} \\
\psi_{-}^{2,6}
\end{array}\right) & =\frac{i}{\sqrt{2}}\left(\begin{array}{cc}
1 & 1 \\
1 & -1
\end{array}\right)\left(\begin{array}{l}
\psi_{-}^{1,6} \\
\psi_{-}^{2,6}
\end{array}\right), \\
T_{(6)-}\left(\begin{array}{c}
\psi_{-}^{1,6} \\
\psi_{-}^{2,6}
\end{array}\right) & =\left(\begin{array}{cc}
e^{\pi i / 6} & 0 \\
0 & e^{2 \pi i / 3}
\end{array}\right)\left(\begin{array}{l}
\psi_{-}^{1,6} \\
\psi_{-}^{2,6}
\end{array}\right) .
\end{aligned}
$$

\section{Non-Abelian discrete flavor symmetries}

In Ref. [8], it is shown that the models with $M=2$ as well as even magnetic fluxes have the $D_{4}$ flavor symmetry. See Appendix A. One of the $Z_{2}$ elements in $D_{4}$ corresponds to $\left(T_{(2)}\right)^{2}$ on the zero modes, $\psi^{0,2}$ and $\psi^{1,2}$, i.e.,

$$
Z=\left(\begin{array}{cc}
1 & 0 \\
0 & -1
\end{array}\right)=\left(T_{(2)}\right)^{2}
$$

In addition, the permutation $Z_{2}^{C}$ element in $D_{4}$ corresponds to $S_{(2)} T_{(2)} T_{(2)} S_{(2)}$, i.e.,

$$
C=\left(\begin{array}{ll}
0 & 1 \\
1 & 0
\end{array}\right)=S_{(2)} T_{(2)} T_{(2)} S_{(2)}
$$

Thus, the $D_{4}$ group, which includes the eight elements (A8), is a subgroup of $G_{(2)} \simeq\left(Z_{8} \times Z_{4}\right) \rtimes S_{3}$.

However, there is the difference between the modular symmetry and the $D_{4}$ flavor symmetry, which is studied in Ref. [8]. The modular symmetry transforms the Yukawa couplings, while the Yukawa couplings are invariant under the $D_{4}$ flavor symmetry. In order to study this point, here we examine the Yukawa couplings among $\psi^{i, 2}, \psi^{\prime j, 2}$, and $\psi^{k, 4}$. Both $\psi^{i, 2}$ and $\psi^{\prime j, 2}$ are $D_{4}$ doublets, and their tensor product $2 \times 2$ is expanded by

$$
\mathbf{2} \times \mathbf{2}=\mathbf{1}_{++}+\mathbf{1}_{+-}+\mathbf{1}_{-+}+\mathbf{1}_{--} .
$$

Thus, the products $\psi^{i, 2} \psi^{\prime j, 2}$ correspond to four singlets, $\mathbf{1}_{+ \pm}: \psi^{0,2} \psi^{\prime 0,2} \pm \psi^{1,2} \psi^{\prime 1,2}, \quad \mathbf{1}_{- \pm}: \psi^{0,2} \psi^{\prime 1,2} \pm \psi^{1,2} \psi^{\prime 0,2}$

On the other hand, by use of Eq. (9), the products $\psi^{i, 2} \psi^{\prime j, 2}$ are expanded by $\psi^{k, 4}$. For example, we can expand as

$$
\begin{aligned}
& \psi^{0,2} \psi^{\prime 0,2} \pm \psi^{1,2} \psi^{\prime 1,2} \sim\left(Y^{(0)}(16 \tau)+Y^{(8 / 16)}(16 \tau)\right. \\
& \left.\quad \pm\left(Y^{(4 / 16)}(16 \tau)+Y^{(12 / 16)}(16 \tau)\right)\right) \\
& \quad \times\left(\psi^{0,4} \pm \psi^{2,4}\right)
\end{aligned}
$$

up to constant factors, where

$$
Y^{(j / M)}(M \tau)=\mathcal{N} \cdot \vartheta\left[\begin{array}{c}
\frac{j}{M} \\
0
\end{array}\right](0, M \tau)
$$

Note that $Y^{(j / M)}(M \tau)=Y^{(1-j / M)}(M \tau)$. It is found that

$$
\begin{aligned}
\left(T_{(4)}\right)^{2}\left(\psi^{0,4} \pm \psi^{2,4}\right) & =\left(\psi^{0,4} \pm \psi^{2,4}\right) \\
\left(S_{(4)} T_{(4)} T_{(4)} S_{(4)}\right)\left(\psi^{0,4} \pm \psi^{2,4}\right) & = \pm\left(\psi^{0,4} \pm \psi^{2,4}\right)
\end{aligned}
$$

Thus, the zero modes $\psi^{0,4} \pm \psi^{2,4}$ are indeed $D_{4}$ singlets, $\mathbf{1}_{+ \pm}$ when we identify $\left(T_{(4)}\right)^{2}$ and $\left(S_{(4)} T_{(4)} T_{(4)} S_{(4)}\right)$ as $Z_{2}$ and $Z_{2}^{C}$ of $D_{4}$. In this sense, the $D_{4}$ flavor symmetry is a subgroup of the modular symmetry. Also, it is found that the above Yukawa couplings, $Y^{(m / 4)}(16 \tau)$, with $m=0,1,2,3$ are invariant under $T^{2}$ and STTS transformation.

Similarly, we can expand

$$
\begin{aligned}
\psi^{0,2} \psi^{\prime 1,2}+\psi^{1,2} \psi^{\prime 0,2} \sim & \left(Y^{(2 / 16)}(16 \tau)+Y^{(6 / 16)}(16 \tau)\right) \\
& \times\left(\psi^{1,4}+\psi^{3,4}\right)
\end{aligned}
$$

up to constant factors. It is found that

$$
\left(T_{(2)}\right)^{2}\left(\psi^{0,2} \psi^{\prime 1,2}+\psi^{1,2} \psi^{\prime 0,2}\right)=-\left(\psi^{0,2} \psi^{\prime 1,2}+\psi^{1,2} \psi^{\prime 0,2}\right) .
$$

On the other hand, we obtain

$$
\left(T_{(4)}\right)^{2}\left(\psi^{1,4}+\psi^{3,4}\right)=i\left(\psi^{1,4}+\psi^{3,4}\right) .
$$

In addition, we find

$$
\begin{aligned}
T^{2}: & \left(Y^{(2 / 16)}(16 \tau)+Y^{(6 / 16)}(16 \tau)\right) \rightarrow i\left(Y^{(2 / 16)}(16 \tau)\right. \\
& \left.+Y^{(6 / 16)}(16 \tau)\right)
\end{aligned}
$$

Thus, the $T^{2}$ transformation is consistent between leftand right-hand sides in (62). However, when we interpret $T^{2}$ as $Z_{2}$ of the $D_{4}$ flavor symmetry, we face inconsistency, because Yukawa couplings are not invariant and $\left(\psi^{1,4}+\right.$ $\left.\psi^{3,4}\right)$ has transformation behavior different from $\left(\psi^{0,2} \psi^{\prime 1,2}+\psi^{1,2} \psi^{\prime 0,2}\right)$. We can make this consistent by 
defining $Z_{2}$ of the $D_{4}$ on $\left(\psi^{1,4}+\psi^{3,4}\right)$ such that its transformation absorbs the phase of Yukawa couplings under $T^{2}$ transformation. Then, the mode $\left(\psi^{1,4}+\psi^{3,4}\right)$ exactly corresponds to the $D_{4}$ singlet, $\mathbf{1}_{-+}$. We find that $\left(\psi^{0,2} \psi^{\prime 1,2}+\psi^{1,2} \psi^{\prime 0,2}\right)$ is invariant under $S_{(2)} T_{(2)} T_{(2)} S_{(2)}$, and $\left(\psi^{1,4}+\psi^{3,4}\right)$ is also invariant under $S_{(4)} T_{(4)} T_{(4)} S_{(4)}$. That is consistent. Therefore, the $D_{4}$ flavor symmetry is a subgroup of the modular symmetry on $\psi^{j, 2}(j=0,1)$. However, when the model includes couplings to zero modes with larger $M$, we have to modify their modular symmetries such that coupling constants are invariant under the flavor symmetry. Then, we can define the $D_{4}$ flavor symmetry.

Here, we give a comment on the $T^{2} / Z_{2}$ orbifold. The $T^{2} / Z_{2}$ orbifold basis gives the irreducible representations of the modular symmetry. The $D_{4}$ flavor symmetry is defined through the modular symmetry, as above. That is the reason why the $D_{4}$ flavor symmetry remains on the $T^{2} / Z_{2}$ orbifold [11,12].

\section{HETEROTIC ORBIFOLD MODELS}

Intersecting D-brane models in type II superstring theory is T-dual to magnetized D-brane models. Thus, intersecting D-brane models also have the same behavior under modular transformation as magnetized D-brane models. Furthermore, intersecting D-brane models in type II superstring theory and heterotic string theory on orbifolds have similarities, e.g., in two-dimensional conformal field theory. For example, computations of three-point couplings as well as $n$-point couplings are similar to each other. Here, we study modular symmetry in heterotic orbifold models. Using results in Refs. [16-18], we compare the modular symmetries in heterotic orbifold models with non-Abelian flavor symmetries and also the modular symmetries in the magnetized D-brane models, which have been derived in the previous section.

\section{A. Twisted sector}

Here, we give a brief review on heterotic string theory on orbifolds. The orbifold is the division of the torus $T^{n}$ by the $Z_{N}$ twist $\theta$, i.e., $T^{n} / Z_{N}$. Since the $T^{n}$ is constructed by $\mathbb{R}^{n} / \Lambda$, the $Z_{N}$ twist $\theta$ should be an automorphism of the lattice $\Lambda$. Here, we focus on two-dimensional orbifolds, $T^{2} / Z_{N}$. The six-dimensional orbifolds can be constructed by products of two-dimensional ones. All of the possible orbifolds are classified as $T^{2} / Z_{N}$ with $N=2,3,4,6$.

On orbifolds, there are fixed points, which satisfy the following condition:

$$
x^{i}=\left(\theta^{n} x\right)^{i}+\sum_{k} m_{k} \alpha_{k}^{i},
$$

where $x^{i}$ are real coordinates, $\alpha_{k}^{i}$ are two lattice vectors, and $m_{k}$ are integers for $i, k=1,2$. Thus, the fixed points can be represented by corresponding space group elements $\left(\theta^{n}, \sum_{k} m_{k} \alpha_{k}^{i}\right)$, or in short $\left(\theta^{n},\left(m_{1}, m_{2}\right)\right)$.

The heterotic string theory on orbifolds has localized modes at fixed points, and these are the so-called twisted strings. These twisted states can be labeled by use of fixed points, $\sigma_{\theta,\left(m_{1}, m_{2}\right)}$. All of the twisted states $\sigma_{\theta,\left(m_{1}, m_{2}\right)}$ have the same spectrum, if discrete Wilson lines vanish. Thus, the massless modes are degenerate by the number of fixed points.

On the $T^{2} / Z_{2}$ orbifold, there are four fixed points, which are denoted by $(\theta,(0,0)),(\theta,(1,0)),(\theta,(0,1)),(\theta,(1,1))$. The corresponding twisted states are denoted by $\sigma_{\theta,(m, n)}$ for $m, n=0,1$.

On the $T^{2} / Z_{3}$ orbifold, $\alpha_{1}$ and $\alpha_{2}$ correspond to the $S U(3)$ simple roots, and they are identified with each other by the $Z_{3}$ twist. Thus, three fixed points on the $T^{2} / Z_{3}$ orbifold are represented by the space group elements, $\left(\theta, m \alpha_{1}\right)$ for $m=0,1,2$, or in short $(\theta, m)$. The corresponding twisted states are denoted by $\sigma_{\theta, m}$ for $m=0,1,2$.

Similarly, we can obtain the fixed points and twisted states on the $T^{2} / Z_{4}$, where $\alpha_{1}$ and $\alpha_{2}$ correspond to the $S O(4)$ simple roots and they are identified with each other by the $Z_{4}$ twist. For the $Z_{4}$ twist $\theta$, two fixed points satisfy Eq. (66), and these can be represented by $\left(\theta, m \alpha_{1}\right)$ for $m=0,1$, or in short $(\theta, m)$. Then, the first twisted states are denoted by $\sigma_{\theta, m}$ for $m=0,1$. In addition, for $\theta^{2}$, there are four points, which satisfy Eq. (66), and these can be denoted by $\left(\theta^{2},(m, n)\right)$ for $m, n=0,1$. Indeed, these correspond to the four fixed points on the $T^{2} / Z_{2}$ orbifold. Then, the second twisted states are denoted by $\sigma_{\theta^{2},(m, n)}$ for $m, n=0,1$. However, the fixed points $\left(\theta^{2},(1,0)\right)$ and $\left(\theta^{2},(0,1)\right)$ transform each other under the $Z_{4}$ twist $\theta$. Thus, the $Z_{4}$ invariant states are written by [21]

$$
\sigma_{\theta^{2},(0,0)}, \quad \sigma_{\theta^{2},+}, \quad \sigma_{\theta^{2},(1,1)},
$$

while $\sigma_{\theta^{2},-}$ transforms to $-\sigma_{\theta^{2},-}$ under the $Z_{4}$ twist, where

$$
\sigma_{\theta^{2}, \pm}=\frac{1}{\sqrt{2}}\left(\sigma_{\theta^{2},(1,0)} \pm \sigma_{\theta^{2},(0,1)}\right) .
$$

Similarly, we can obtain the fixed points on $T^{2} / Z_{6}$. There is a fixed point $(\theta, 0)$ for the $Z_{6}$ twist $\theta$, and a single twisted state $\sigma_{\theta, 0}$. The second twisted sector has three fixed points $\left(\theta^{2}, m\right)(m=0,1,2)$, which correspond to the three fixed points on the $T^{2} / Z_{3}$ orbifold. The two fixed points $\left(\theta^{2}, 1\right)$ and $\left(\theta^{2}, 2\right)$ transform each other by the $Z_{6}$ twist, while $\left(\theta^{2}, 0\right)$ is invariant. Thus, we can write the $Z_{6}$-invariant $\theta^{2}$-twisted states by

$$
\sigma_{\theta^{2}, 0}, \quad \sigma_{\theta^{2},+},
$$

while $\sigma_{\theta^{2},-}$ transforms to $-\sigma_{\theta^{2},-}$ under the $Z_{6}$ twist, where

$$
\sigma_{\theta^{2}, \pm}=\frac{1}{\sqrt{2}}\left(\sigma_{\theta^{2}, 1} \pm \sigma_{\theta^{2}, 2}\right) .
$$


The third twisted sector has four fixed points, which correspond to the fixed points on $T^{2} / Z_{2}$, and the corresponding $\theta^{3}$ twisted states. Their linear combinations are $Z_{6}$ eigenstates similar to the second twisted states. Since the first twisted sector has the single fixed point and twisted state, the modular symmetry as well as non-Abelian discrete flavor symmetry is rather trivial. We do not discuss the $T^{2} / Z_{6}$ orbifold itself.

\section{B. Modular symmetry}

In Ref. [16], modular symmetry in heterotic string theory on orbifolds was studied in detail. Here we use those results.

\section{1. $T^{2} / Z_{4}$ orbifold}

The $S$ and $T$ transformations are represented by the first twisted sectors of $T^{2} / Z_{4}$ orbifold as [16]

$$
\begin{aligned}
\left(\begin{array}{c}
\sigma_{\theta, 0} \\
\sigma_{\theta, 1}
\end{array}\right) \rightarrow S_{Z_{4}}\left(\begin{array}{c}
\sigma_{\theta, 0} \\
\sigma_{\theta, 1}
\end{array}\right), & S_{Z_{4}}=\frac{1}{\sqrt{2}}\left(\begin{array}{cc}
1 & 1 \\
1 & -1
\end{array}\right), \\
\left(\begin{array}{c}
\sigma_{\theta, 0} \\
\sigma_{\theta, 1}
\end{array}\right) \rightarrow T_{Z_{4}}\left(\begin{array}{c}
\sigma_{\theta, 0} \\
\sigma_{\theta, 1}
\end{array}\right), & T_{Z_{4}}=\left(\begin{array}{ll}
1 & 0 \\
0 & i
\end{array}\right) .
\end{aligned}
$$

These are exactly the same as representations of $S_{(2)}$ and $T_{(2)}$ on two-zero modes, $\psi^{0,2}$ and $\psi^{1,2}$ in the magnetized model with magnetic flux $M=2$. Hence, the twisted sectors on the $T^{2} / Z_{4}$ orbifold has the same behavior of modular symmetry as the magnetized model with magnetic flux $M=2$. Indeed, the twisted sectors have the $D_{4}$ flavor symmetry and two twisted states, $\sigma_{\theta, 0}$ and $\sigma_{\theta, 1}$, correspond to the $D_{4}$ doublet [4]. The whole flavor symmetry of the $T^{2} / Z_{4}$ orbifold model is slightly larger than $D_{4}$. (See Appendix B.) The $T^{2} / Z_{4}$ orbifold model has the $Z_{4}$ symmetry, which transforms the first twisted sector,

$$
\sigma_{\theta, m} \rightarrow e^{\pi i / 2} \sigma_{\theta, m},
$$

for $m=0,1$ and the second twisted sector,

$$
\sigma_{\theta^{2},(m, n)} \rightarrow e^{\pi i} \sigma_{\theta^{2},(m, n)},
$$

for $m, n=0,1$. The above $Z_{4}$ transformation (72) is nothing but $\left(S_{Z_{4}} T_{Z_{4}}\right)^{6}$ as clearly seen from Eq. (24). Thus, the whole flavor symmetry originates from the modular symmetry.

The second twisted sectors correspond to $D_{4}$ singlets, $\mathbf{1}_{ \pm 1, \pm}[4]$, as

$$
\mathbf{1}_{+ \pm}: \sigma_{\theta^{2},(0,0)} \pm \sigma_{\theta^{2},(1,1)}, \quad \mathbf{1}_{- \pm}: \sigma_{\theta^{2}, \pm},
$$

up to coefficients. Compared with the results in Sec. II C, the $D_{4}$ behavior of the second twisted states corresponds to one of the zero modes $\psi^{m, 4}$ with magnetic flux $M=4$. Their correspondence can be written as

$$
\begin{aligned}
\sigma_{\theta^{2},(0,0)} & \sim \psi^{0,4}, & & \sigma_{\theta^{2},(1,1)} \sim \psi^{2,4}, \\
\sigma_{\theta^{2},(1,0)} & \sim \psi^{1,4}, & & \sigma_{\theta^{2},(1,0)} \sim \psi^{3,4} .
\end{aligned}
$$

The above correspondence can also been seen from the Yukawa couplings. By use of operator product expansion, we obtain the following relations [16]:

$$
\begin{gathered}
\sigma_{\theta, 0} \sigma_{\theta, 0} \sim Y_{0,0}\left(\sigma_{\theta^{2},(0,0)}+\sigma_{\theta^{2},(1,1)}\right), \\
\sigma_{\theta, 1} \sigma_{\theta, 1} \sim Y_{1,1}\left(\sigma_{\theta^{2},(0,0)}+\sigma_{\theta^{2},(1,1)}\right), \\
\sigma_{\theta, 0} \sigma_{\theta, 1}+\sigma_{\theta, 1} \sigma_{\theta, 0} \sim Y_{0,1} \sigma_{\theta^{2},+}
\end{gathered}
$$

up to constants. The second twisted state $\sigma_{\theta^{2},-}$ cannot couple with the first twisted sectors. Using results in Ref. [16], it is found that

$$
\left(T_{Z_{4}}\right)^{2}\left(\begin{array}{l}
Y_{0,0} \\
Y_{1,1} \\
Y_{0,1}
\end{array}\right)=\left(\begin{array}{ccc}
1 & 0 & 0 \\
0 & 1 & 0 \\
0 & 0 & -1
\end{array}\right)\left(\begin{array}{l}
Y_{0,0} \\
Y_{1,1} \\
Y_{0,1}
\end{array}\right) .
$$

This is the same as behavior of the Yukawa couplings under $T^{2}$ studied in Sec. II C.

\section{2. $T^{2} / Z_{2}$ orbifold}

Here, let us study the $T^{2} / Z_{2}$ orbifold in a way similar to the previous section on the $T^{2} / Z_{4}$. The $S$ transformation is represented by the four twisted states on the $T^{2} / Z_{2}$ orbifold [16],

$$
\begin{aligned}
\left(\begin{array}{l}
\sigma_{\theta,(0,0)} \\
\sigma_{\theta,(0,1)} \\
\sigma_{\theta,(1,0)} \\
\sigma_{\theta,(1,1)}
\end{array}\right) \rightarrow S_{Z_{2}}\left(\begin{array}{c}
\sigma_{\theta,(0,0)} \\
\sigma_{\theta,(0,1)} \\
\sigma_{\theta,(1,0)} \\
\sigma_{\theta,(1,1)}
\end{array}\right), \\
S_{Z_{2}}=\frac{1}{2}\left(\begin{array}{cccc}
1 & 1 & 1 & 1 \\
1 & -1 & 1 & -1 \\
1 & 1 & -1 & -1 \\
1 & -1 & -1 & 1
\end{array}\right) .
\end{aligned}
$$

Also the $T$ transformation is represented as

$$
\begin{aligned}
\left(\begin{array}{c}
\sigma_{\theta,(0,0)} \\
\sigma_{\theta,(0,1)} \\
\sigma_{\theta,(1,0)} \\
\sigma_{\theta,(1,1)}
\end{array}\right) & \rightarrow T_{Z_{2}}\left(\begin{array}{c}
\sigma_{\theta,(0,0)} \\
\sigma_{\theta,(0,1)} \\
\sigma_{\theta,(1,0)} \\
\sigma_{\theta,(1,1)}
\end{array}\right), \\
T_{Z_{2}} & =\left(\begin{array}{cccc}
1 & 0 & 0 & 0 \\
0 & -1 & 0 & 0 \\
0 & 0 & -1 & 0 \\
0 & 0 & 0 & -1
\end{array}\right) .
\end{aligned}
$$

The representation $S_{Z_{2}}$ is similar to $S_{Z_{4}}$ and $S_{(2)}$. Indeed, we find that $S_{Z_{2}}=S_{(2)} \otimes S_{(2)}$. However, the representation $T_{Z_{2}}$ is different from $T_{Z_{4}}$ and $T_{(2)}$. 
The matrices $S_{Z_{2}}$ and $T_{Z_{2}}$ satisfy the following relations:

$$
\left(S_{Z_{2}}\right)^{2}=\left(T_{Z_{2}}\right)^{2}=\left(S_{Z_{2}} T_{Z_{2}}\right)^{6}=\mathbb{I} \text {. }
$$

These correspond to the $D_{6}$. Indeed, the order of closed algebra including $S_{Z_{2}}$ and $T_{Z_{2}}$ is equal to 12 . At any rate, these matrices are reducible. We change the basis in order to obtain irreducible representations,

$$
\left(\begin{array}{l}
\sigma_{1} \\
\sigma_{2} \\
\sigma_{3} \\
\sigma_{4}
\end{array}\right)=\left(\begin{array}{cccc}
1 & 0 & 0 & 0 \\
0 & \frac{1}{\sqrt{3}} & \frac{1}{\sqrt{3}} & \frac{1}{\sqrt{3}} \\
0 & \frac{1}{\sqrt{2}} & \frac{-1}{\sqrt{3}} & 0 \\
0 & \frac{1}{\sqrt{6}} & \frac{1}{\sqrt{6}} & \frac{-2}{\sqrt{6}}
\end{array}\right)\left(\begin{array}{c}
\sigma_{\theta,(0,0)} \\
\sigma_{\theta,(1,0)} \\
\sigma_{\theta,(0,1)} \\
\sigma_{\theta,(1,1)}
\end{array}\right) .
$$

Then, $\sigma_{1}$ and $\sigma_{2}$ correspond to the $D_{6}$ doublet, while $\sigma_{3}$ and $\sigma_{4}$ correspond to the $D_{6}$ singlets. For example, $S_{Z_{2}} T_{Z_{2}}$ and $T_{Z_{2}}$ are represented by

$$
\begin{aligned}
S_{Z_{2}} T_{Z_{2}}\left(\begin{array}{l}
\sigma_{1} \\
\sigma_{2} \\
\sigma_{3} \\
\sigma_{4}
\end{array}\right)= & \left(\begin{array}{cccc}
\cos (2 \pi / 6) & -\sin (2 \pi / 6) & 0 & 0 \\
\sin (2 \pi / 6) & \cos (2 \pi / 6) & 0 & 0 \\
0 & 0 & 1 & 0 \\
0 & 0 & 0 & -1
\end{array}\right) \\
& \times\left(\begin{array}{c}
\sigma_{1} \\
\sigma_{2} \\
\sigma_{3} \\
\sigma_{4}
\end{array}\right), \\
T_{Z_{2}}\left(\begin{array}{l}
\sigma_{1} \\
\sigma_{2} \\
\sigma_{3} \\
\sigma_{4}
\end{array}\right)= & \left(\begin{array}{cccc}
1 & 0 & 0 & 0 \\
0 & -1 & 0 & 0 \\
0 & 0 & -1 & 0 \\
0 & 0 & 0 & -1
\end{array}\right)\left(\begin{array}{l}
\sigma_{1} \\
\sigma_{2} \\
\sigma_{3} \\
\sigma_{4}
\end{array}\right) .
\end{aligned}
$$

It is found that $\sigma_{3}$ and $\sigma_{4}$ correspond to $\mathbf{1}_{--}$and $\mathbf{1}_{-+}$.

The twisted sector on the $T^{2} / Z_{2}$ orbifold has the flavor symmetry $\left(D_{4} \times D_{4}\right) / Z_{2}$. However, this flavor symmetry seems independent of the above $D_{6}$, because they do not include any common elements. The twisted sector on the $S^{1} / Z_{2}$ orbifold has the flavor symmetry $D_{4}$. The flavor symmetry of the $T^{2} / Z_{2}$ orbifold is obtained as a kind of product, $D_{4} \times D_{4}$, although two $D_{4}$ groups have a common $Z_{2}$ element. Thus, the flavor symmetry of $T^{2} / Z_{2}$ originates from the product of symmetries of the onedimensional orbifold. On the other hand, the modular symmetry appears in two or more dimensions, but not in one dimension. Hence, these symmetries would be independent. When we include the above $D_{6}$ as low-energy effective field theory in addition to the flavor symmetry $\left(D_{4} \times D_{4}\right) / Z_{2}$, low-energy effective field theory would have larger symmetry including $D_{6}$ and $\left(D_{4} \times D_{4}\right) / Z_{2}$, although Yukawa couplings as well as higher order couplings transform nontrivially under $D_{6}$.

\section{3. $T^{2} / Z_{3}$ orbifold}

The $S$ and $T$ transformations are represented by the first twisted sectors of the $T^{2} / Z_{3}$ orbifold as [16]

$$
\begin{aligned}
\left(\begin{array}{c}
\sigma_{\theta, 0} \\
\sigma_{\theta, 1} \\
\sigma_{\theta, 2}
\end{array}\right) & \rightarrow S_{Z_{3}}\left(\begin{array}{c}
\sigma_{\theta, 0} \\
\sigma_{\theta, 1} \\
\sigma_{\theta, 2}
\end{array}\right), \\
S_{Z_{3}} & =\frac{1}{\sqrt{3}}\left(\begin{array}{ccc}
1 & 1 & 1 \\
1 & e^{2 \pi i / 3} & e^{-2 \pi i / 3} \\
1 & e^{-2 \pi i / 3} & e^{2 \pi i / 3}
\end{array}\right), \\
\left(\begin{array}{c}
\sigma_{\theta, 0} \\
\sigma_{\theta, 1} \\
\sigma_{\theta, 2}
\end{array}\right) & \rightarrow T_{Z_{3}}\left(\begin{array}{cc}
\sigma_{\theta, 0} \\
\sigma_{\theta, 1} \\
\sigma_{\theta, 2}
\end{array}\right), \\
T_{Z_{3}} & =\left(\begin{array}{ccc}
1 & 0 & 0 \\
0 & e^{2 \pi i / 3} & 0 \\
0 & 0 & e^{2 \pi i / 3}
\end{array}\right) .
\end{aligned}
$$

These forms look similar to $S$ and $T$ transformations in magnetized models (18) and (19). Indeed, they correspond to submatrices of $S_{(6)}$ and $T_{(6)}$ in the magnetized models with the magnetic flux $M=6$. Alternatively, in Ref. [17] the following $S$ and $T$ representations were studied ${ }^{3}$

$$
\begin{aligned}
S_{Z_{3}}^{\prime} & =-\frac{i}{\sqrt{3}}\left(\begin{array}{ccc}
1 & 1 & 1 \\
1 & e^{2 \pi i / 3} & e^{-2 \pi i / 3} \\
1 & e^{-2 \pi i / 3} & e^{2 \pi i / 3}
\end{array}\right), \\
T_{Z_{3}}^{\prime} & =\left(\begin{array}{ccc}
e^{2 \pi i / 3} & 0 & 0 \\
0 & 1 & 0 \\
0 & 0 & 1
\end{array}\right) .
\end{aligned}
$$

At any rate, the above representations are reducible representations. Thus, we use the flowing basis,

$$
\left(\begin{array}{c}
\sigma_{+} \\
\sigma_{0} \\
\sigma_{-}
\end{array}\right),
$$

where $\sigma_{ \pm}=\left(\sigma_{1} \pm \sigma_{-}\right) / \sqrt{2}$. The $\left(\sigma_{+}, \sigma_{0}\right)$ is a doublet, while $\sigma_{-}$is a singlet. The former corresponds to the $Z_{6}$ invariant states among the $\theta^{2}$ twisted sector on the $T^{2} / Z_{6}$ orbifold. Similarly, $\sigma_{-}$is the $\theta^{2}$ twisted state, which transforms $\sigma_{-} \rightarrow-\sigma_{-}$under the $Z_{6}$ twist. Alternatively, we can say that the doublet $\left(\sigma_{+}, \sigma_{0}\right)$ corresponds to $Z_{2}$ even states and the singlet $\sigma_{-}$is the $Z_{2}$ odd states, where the $Z_{2}$ means the $\pi$ rotation of the lattice vectors, $\left(\alpha_{1}, \alpha_{2}\right) \rightarrow$ $\left(-\alpha_{1},-\alpha_{2}\right)$. This point is similar to the aspect in magnetized D-brane models, where irreducible representations

\footnotetext{
${ }^{3}$ See also Ref. [18].
} 
correspond to the $T^{2} / Z_{2}$ orbifold basis. Also, note that the first twisted states of the $T^{2} / Z_{4}$ orbifold correspond already to the $Z_{2}$-invariant basis.

For example, we represent $S_{Z_{3}}^{\prime}$ and $T_{Z_{3}}^{\prime}$ on the above basis [17],

$S_{Z_{3}}^{\prime}=\frac{i}{\sqrt{3}}\left(\begin{array}{cc}1 & \sqrt{2} \\ \sqrt{2} & -1\end{array}\right), \quad T_{Z_{3}}^{\prime}=\left(\begin{array}{cc}e^{2 \pi i / 3} & 0 \\ 0 & 1\end{array}\right)$,

on the doublet $\left(\sigma_{+}, \sigma_{0}\right)^{T}$, while $\sigma_{-}$is the trivial singlet. Here, we define

$$
Z=\left(\begin{array}{cc}
-1 & 0 \\
0 & -1
\end{array}\right), \quad \tilde{T}_{Z_{3}}=Z T_{Z_{3}}^{\prime}
$$

Then, they satisfy the following algebraic relations $[17,18]$ :

$$
\left(S_{Z_{3}}^{\prime}\right)^{2}=\left(\tilde{T}_{Z_{3}}\right)^{3}=\left(S_{Z_{3}}^{\prime} \tilde{T}_{Z_{3}}\right)^{3}=Z, \quad Z^{2}=\mathbb{I} .
$$

This group is the so-called $T^{\prime}$, which is the binary extension of $A_{4}=T$.

The non-Abelian discrete flavor symmetry on the $T^{2} / Z_{3}$ orbifold is $\Delta(54)$, and the three twisted states correspond to the triplet of $\Delta(54)$. Thus, this modular symmetry seems independent of the $\Delta(54)$ flavor symmetry.

Two representations are related as

$$
S_{Z_{3}}^{\prime}=-i S_{Z_{3}}, \quad T_{Z_{3}}^{\prime}=e^{2 \pi i / 3}\left(T_{Z_{3}}\right)^{-1} .
$$

When we change phases of $S, T$, and $S T$, the group such as $\left(Z_{N} \times Z_{M}\right) \rtimes H$ in Secs. II and III would change to $\left(Z_{N^{\prime}} \times Z_{M^{\prime}}\right) \rtimes H$.

\section{CONCLUSION}

We have studied the modular symmetries in magnetized D-brane models. Representations due to zero modes on $T^{2}$ are reducible except the models with the magnetic flux $M=2$. Irreducible representations are provided by zero modes on the $T^{2} / Z_{2}$, i.e., $Z_{2}$ even states and odd states. It is reasonable because $(S T)^{3}$ transforms the lattice vectors $\left(\alpha_{1}, \alpha_{2}\right)$ to $\left(-\alpha_{1},-\alpha_{2}\right)$. The orders of modular groups are large, and in general, they include the $Z_{8}$ symmetry as the center. The $D_{4}$ flavor symmetry is a subgroup of the modular group, which is represented in the models with the magnetic flux $M=2$. The system including zero modes with $M=2, M=4$, and larger even $M$, also includes the $D_{4}$ flavor symmetry, when we define transformations of couplings in a proper way.

We have also studied the modular symmetries in heterotic orbifold models. The heterotic model on the $T^{2} / Z_{4}$ has exactly the same representation as the magnetized model with $M=2$, and the modular symmetry includes the $D_{4}$ flavor symmetry. The representation due to the twisted states on the $T^{2} / Z_{3}$ orbifold is reducible, similar to representations due to zero modes in magnetized D-brane models on $T^{2}$. Their irreducible representations correspond to $Z_{2}$ even and odd states, similar to those in magnetized D-brane models. Thus, the $\Delta(54)$ flavor symmetry seems independent of the modular symmetry in the $T^{2} / Z_{3}$ orbifold models. Note that the first twisted states on the $T^{2} / Z_{4}$ are $Z_{2}$-invariant states. In this sense, we find that the modular symmetry is the symmetry on the $Z_{2}$ orbifold in both heterotic orbifold models and magnetized D-brane models. The symmetries, which remain under the $Z_{2}$ twist, can be realized as the modular symmetry.

We have set vanishing Wilson lines. It would be interesting to extend our analysis to magnetized D-brane models with discrete Wilson lines on orbifolds [22]. It would also be interesting to extend our analysis on zero modes to higher Kaluza-Klein modes [23].

Four-dimensional low energy-effective field theory is modular invariant [24-26]. Anomalies of the modular symmetry were studied $[27,28]$, and they have important aspects [29-31]. The non-Abelian flavor symmetries such as $D_{4}$ can be anomalous. (See for anomalies of non-Abelian discrete symmetries, e.g., $[2,32,33]$.) In certain models, the modular symmetries are related with the non-Abelian flavor symmetry $D_{4}$. It would be interesting to study their anomaly relations.

We also give a comment on phenomenological application. Recently, the mixing angles in the lepton sector were studied in the models, whose flavor symmetries are congruence subgroups, $\Gamma(N)$ [34,35]. In those models, the couplings are nontrivial representations of $\Gamma(N)$ and modular functions. Our models show massless modes represent larger finite groups. It would be interesting to apply our results to derive realistic lepton mass matrices as well as quark mass matrices.

\section{ACKNOWLEDGMENTS}

T. K. is supported in part by MEXT KAKENHI Grant No. JP17H05395 and JSP KAKENHI Grant No. JP26247042.

\section{APPENDIX A: NON-ABELIAN DISCRETE FLAVOR SYMMETRY IN MAGNETIZED D-BRANE MODELS}

In this appendix, we give a brief review on non-Abelian discrete flavor symmetries in magnetized D-brane models [8].

As mentioned in Sec. II A, the Yukawa couplings as well as higher order couplings have the coupling selection rule (11). That is, we can define $Z_{g}$ charges for zero modes. Such $Z_{g}$ transformation is represented on $\psi^{i, M=g}$ by 


$$
Z=\left(\begin{array}{ccccc}
1 & & & & \\
& \rho & & & \\
& & \rho^{2} & & \\
& & & \ddots & \\
& & & & \rho^{g-1}
\end{array}\right),
$$

where $\rho=e^{2 \pi i / g}$. Furthermore, their effective field theory has the following permutation symmetry:

$$
\psi^{i, g} \rightarrow \psi^{i+1, g},
$$

and such permutation can be represented by

$$
C=\left(\begin{array}{cccccc}
0 & 1 & 0 & 0 & \cdots & 0 \\
0 & 0 & 1 & 0 & \cdots & 0 \\
& & & & \ddots & \\
1 & 0 & 0 & 0 & \cdots & 0
\end{array}\right) .
$$

This is another $Z_{g}^{C}$ symmetry. However, these two generators do not commute with each other,

$$
C Z=\rho Z C
$$

Thus, the flavor symmetry corresponds to the closed algebra including $Z$ and $C$. Its diagonal elements are given by $Z^{m} Z^{\prime n}$, i.e., $Z_{g} \times Z_{g}^{\prime}$ where

$$
Z^{\prime}=\left(\begin{array}{lll}
\rho & & \\
& \ddots & \\
& & \rho
\end{array}\right),
$$

and the full group corresponds to $\left(Z_{g} \times Z_{g}^{\prime}\right) \rtimes Z_{g}^{C}$.

Furthermore, the zero modes $\psi^{i, M=g n}$ with the magnetic flux $M=g n$ also represent $\left(Z_{g} \times Z_{g}^{\prime}\right) \rtimes Z_{g}^{C}$. The zero modes, $\psi^{i, M=g n}$, have $Z_{g}$ charges $(\bmod g)$. Under $C$, they transform as

$$
\psi^{i, M=g n} \rightarrow \psi^{i+n, M=g n} .
$$

For example, the model with $g=2$ has the $D_{4}$ flavor symmetry. The zero modes,

$$
\left(\begin{array}{l}
\psi^{0,2} \\
\psi^{1,2}
\end{array}\right)
$$

correspond to the $D_{4}$ doublet 2 , where eight $D_{4}$ elements are represented by
TABLE I. $\quad D_{4}$ representation.

\begin{tabular}{lc}
\hline \hline Magnetic flux $M$ & $D_{4}$ representations \\
\hline 2 & $\mathbf{1}_{++}, \underset{+}{\mathbf{2}}, \mathbf{1}_{-+}, \mathbf{1}_{--}$ \\
4 & \\
6 & \\
\hline \hline$\left(\begin{array}{cc}1 & 0 \\
0 & 1\end{array}\right), \quad \pm\left(\begin{array}{ll}0 & 1 \\
1 & 0\end{array}\right)$, & $\pm\left(\begin{array}{cc}0 & 1 \\
-1 & 0\end{array}\right)$, \\
$\pm\left(\begin{array}{cc}1 & 0 \\
0 & -1\end{array}\right)$. &
\end{tabular}

In addition, when the model has the zero modes $\psi^{i, 4}(i=0$, $1,2,3)$, the zero modes $\psi^{0,4}$ and $\psi^{2,4}\left(\psi^{1,4}\right.$ and $\left.\psi^{3,4}\right)$ transform each other under $C$, and they have $Z_{2}$ charge even (odd). Thus, $\psi^{0,4} \pm \psi^{2,4}$ correspond to $\mathbf{1}_{+ \pm}$of $D_{4}$ representations, while $\psi^{1,4} \pm \psi^{3,4}$ correspond to $\mathbf{1}_{- \pm}$. Furthermore, among the zero modes $\psi^{i, 6}(i=0,1,2,3$, 4,5 ), the zero modes $\psi^{i, 6}$ and $\psi^{i+3,6}$ transform each other under $C$. Hence, three pairs of zero modes,

$$
\left(\begin{array}{l}
\psi^{0,6} \\
\psi^{3,6}
\end{array}\right), \quad\left(\begin{array}{l}
\psi^{1,6} \\
\psi^{4,6}
\end{array}\right), \quad\left(\begin{array}{l}
\psi^{2,6} \\
\psi^{5,6}
\end{array}\right),
$$

correspond to three $D_{4}$ doublets. These results are shown in Table I.

\section{APPENDIX B: NON-ABELIAN DISCRETE FLAVOR SYMMETRY IN HETEROTIC ORBIFOLD MODELS}

Here, we give a brief review on non-Abelian discrete flavor symmetries in heterotic orbifold models [4].

The twisted string $x^{i}$ on the orbifold satisfy the following boundary condition:

$$
x^{i}(\sigma=2 \pi)=\left(\theta^{n} x(\sigma=0)\right)^{i}+\sum_{k} m_{k} \alpha_{k}^{i},
$$

similar to Eq. (66). Thus, the twisted string can be characterized by the space group element $g=$ $\left(\theta^{n}, \sum_{k} m_{k} \alpha_{k}^{i}\right)$. The product of the two space group elements $\left(\theta^{n_{1}}, v_{1}\right)$ and $\left(\theta^{n_{2}}, v_{2}\right)$ is computed as

$$
\left(\theta^{n_{1}}, v_{1}\right)\left(\theta^{n_{2}}, v_{2}\right)=\left(\theta^{n_{1}} \theta^{n_{2}}, v_{1}+\theta^{n_{1}} v_{2}\right) .
$$

The space group element $g$ belongs to the same conjugacy class as $h g h^{-1}$, where $h$ is any space group element on the same orbifold.

Now, let us consider the couplings among twisted strings corresponding to space group elements $\left(\theta^{n_{k}}, v_{k}\right)$. Their couplings are allowed by the space group invariance if the following condition,

$$
\prod_{k}\left(\theta^{n_{k}}, v_{k}\right)=(1,0)
$$


is satisfied up to the conjugacy class. That includes the point group selection rule, $\prod_{k} \theta^{n_{k}}=1$, which is the $Z_{N}$ invariance on the $Z_{N}$ orbifold. We can define discrete Abelian symmetries from the space group invariance as well as the point group invariance. These symmetries together with geometrical symmetries of orbifolds become non-Abelian discrete flavor symmetries in heterotic orbifold models. We show them explicitly on concrete orbifolds.

\section{1. $S^{1} / Z_{2}$ orbifold}

The $S^{1} / Z_{2}$ orbifold has two fixed points, which are denoted by the space group elements, $(\theta, m \alpha)$ with $m=0,1$, where $\alpha$ is the lattice vector. In short, we denote them by $(\theta, m)$ and the corresponding twisted states are denoted by $\sigma_{(\theta, m)}$. These states transform

$$
\left(\begin{array}{c}
\sigma_{\theta, 0} \\
\sigma_{\theta, 1}
\end{array}\right) \rightarrow\left(\begin{array}{cc}
-1 & 0 \\
0 & -1
\end{array}\right)\left(\begin{array}{c}
\sigma_{\theta, 0} \\
\sigma_{\theta, 1}
\end{array}\right)
$$

under the $Z_{2}$ twist. In addition, the space group invariance requires $\sum_{k} m_{k}=0(\bmod 2)$ for the couplings corresponding to the product of the space group elements $\prod_{k}\left(\theta, m_{k}\right)$ with $m_{k}=0,1$. Hence, we can define another $Z_{2}$ symmetry, under which $\sigma_{(\theta, 0)}$ is even, while $\sigma_{(\theta, 1)}$ is odd. That is, another $Z_{2}$ transformation can be written by

$$
\left(\begin{array}{c}
\sigma_{\theta, 0} \\
\sigma_{\theta, 1}
\end{array}\right) \rightarrow\left(\begin{array}{cc}
1 & 0 \\
0 & -1
\end{array}\right)\left(\begin{array}{c}
\sigma_{\theta, 0} \\
\sigma_{\theta, 1}
\end{array}\right)
$$

Furthermore, there is the geometrical permutation symmetry, which exchanges two fixed points with each other. Such a permutation is represented by

$$
\left(\begin{array}{c}
\sigma_{\theta, 0} \\
\sigma_{\theta, 1}
\end{array}\right) \rightarrow\left(\begin{array}{ll}
0 & 1 \\
1 & 0
\end{array}\right)\left(\begin{array}{c}
\sigma_{\theta, 0} \\
\sigma_{\theta, 1}
\end{array}\right) .
$$

The closed algebra including Eqs. (B4), (B5), and (B6) is $D_{4} \simeq\left(Z_{2} \times Z_{2}\right) \rtimes Z_{2}$.

\section{2. $T^{2} / Z_{3}$ orbifold}

As shown in Sec. III, the $T^{2} / Z_{3}$ orbifold has three fixed points denoted by $(\theta, m)$ with $m=0,1,2$, and the corresponding twisted states are denoted by $\sigma_{(\theta, m)}$. The $Z_{3}$ twist transforms

$$
\left(\begin{array}{c}
\sigma_{\theta, 0} \\
\sigma_{\theta, 1} \\
\sigma_{\theta, 2}
\end{array}\right) \rightarrow\left(\begin{array}{ccc}
e^{2 \pi i / 3} & 0 & 0 \\
0 & e^{2 \pi i / 3} & 0 \\
0 & 0 & e^{2 \pi i / 3}
\end{array}\right)\left(\begin{array}{c}
\sigma_{\theta, 0} \\
\sigma_{\theta, 1} \\
\sigma_{\theta, 2}
\end{array}\right) .
$$

The space group invariance requires $\sum_{k} m_{k}=0(\bmod 3)$ for the couplings corresponding to the product of the space group elements $\prod_{k}\left(\theta, m_{k}\right)$ with $m_{k}=0,1,2$. Then, we can define another $Z_{3}$ symmetry, under which $\sigma_{(\theta, m)}$ transform

$$
\left(\begin{array}{c}
\sigma_{\theta, 0} \\
\sigma_{\theta, 1} \\
\sigma_{\theta, 2}
\end{array}\right) \rightarrow\left(\begin{array}{ccc}
1 & 0 & 0 \\
0 & e^{2 \pi i / 3} & 0 \\
0 & 0 & e^{2 \pi i / 3}
\end{array}\right)\left(\begin{array}{c}
\sigma_{\theta, 0} \\
\sigma_{\theta, 1} \\
\sigma_{\theta, 2}
\end{array}\right) .
$$

There is also the permutation symmetry of the three fixed points, that is, $S_{3}$. Thus, the flavor symmetry is $\Delta(54) \simeq$ $\left(Z_{3} \times Z_{3}\right) \rtimes S_{3}$.

\section{3. $T^{2} / Z_{4}$ orbifold}

As shown in Sec. III, the $T^{2} / Z_{4}$ orbifold has two $\theta$ fixed points denoted by $(\theta, m)$ with $m=0,1$, and the corresponding twisted states are denoted by $\sigma_{(\theta, m)}$. The $Z_{4}$ twist transforms

$$
\left(\begin{array}{c}
\sigma_{\theta, 0} \\
\sigma_{\theta, 1}
\end{array}\right) \rightarrow\left(\begin{array}{cc}
i & 0 \\
0 & i
\end{array}\right)\left(\begin{array}{c}
\sigma_{\theta, 0} \\
\sigma_{\theta, 1}
\end{array}\right)
$$

The space group invariance requires $\sum_{k} m_{k}=0(\bmod 2)$ for the couplings corresponding to the product of the space group elements $\prod_{k}\left(\theta, m_{k}\right)$ with $m_{k}=0,1$. Then, we can define another $Z_{2}$ symmetry, under which $\sigma_{(\theta, m)}$ transform

$$
\left(\begin{array}{c}
\sigma_{\theta, 0} \\
\sigma_{\theta, 1} \\
\sigma_{\theta, 2}
\end{array}\right) \rightarrow\left(\begin{array}{cc}
1 & 0 \\
0 & -1
\end{array}\right)\left(\begin{array}{c}
\sigma_{\theta, 0} \\
\sigma_{\theta, 1}
\end{array}\right) \text {. }
$$

There is also the permutation symmetry of the two fixed points. Thus, the flavor symmetry is almost the same as the one on the $S^{1} / Z_{2}$ orbifold. The difference is the $Z_{4}$ twist, although its squire is nothing but the $Z_{2}$ twist. Hence, the flavor symmetry can be written as $\left(D_{4} \times Z_{4}\right) / Z_{2}$.

\section{4. $T^{2} / Z_{2}$ orbifold}

As shown in Sec. III, the $T^{2} / Z_{4}$ orbifold has two $\theta$ fixed points denoted by $(\theta,(m, n))$ with $m, n=0,1$, and the corresponding twisted states are denoted by $\sigma_{\theta,(m, n)}$. The space group invariance requires $\sum_{k} m_{k}=\sum_{j} n_{j}=0(\bmod 2)$ for the couplings corresponding to the product of the space group elements $\prod_{k}\left(\theta,\left(m_{k}, n_{j}\right)\right)$ with $m_{k}, n_{j}=0,1$. There are two independent permutation symmetries between $(\theta,(0, n))$ and $(\theta,(1, n))$, and $(\theta,(m, 0))$ and $(\theta,(m, 1))$. Thus, this structure seems be a product of two onedimensional orbifolds, $S^{1} / Z_{2}$. However, the $Z_{2}$ twist is commented such as $\sigma_{\theta,(m, n)} \rightarrow-\sigma_{\theta,(m, n)}$. Thus, the flavor symmetry can be written by $\left(D_{4} \times D_{4}\right) / Z_{2}$. 
[1] G. Altarelli and F. Feruglio, Rev. Mod. Phys. 82, 2701 (2010).

[2] H. Ishimori, T. Kobayashi, H. Ohki, Y. Shimizu, H. Okada, and M. Tanimoto, Prog. Theor. Phys. Suppl. 183, 1 (2010); Lect. Notes Phys. 858, 1 (2012).

[3] S. F. King and C. Luhn, Rep. Prog. Phys. 76, 056201 (2013).

[4] T. Kobayashi, H. P. Nilles, F. Ploger, S. Raby, and M. Ratz, Nucl. Phys. B768, 135 (2007).

[5] T. Kobayashi, S. Raby, and R. J. Zhang, Nucl. Phys. B704, 3 (2005).

[6] P. Ko, T. Kobayashi, J. h. Park, and S. Raby, Phys. Rev. D 76, 035005 (2007); 76, 059901(E) (2007).

[7] F. Beye, T. Kobayashi, and S. Kuwakino, Phys. Lett. B 736, 433 (2014).

[8] H. Abe, K. S. Choi, T. Kobayashi, and H. Ohki, Nucl. Phys. B820, 317 (2009).

[9] H. Abe, K. S. Choi, T. Kobayashi, and H. Ohki, Phys. Rev. D 80, 126006 (2009); 81, 126003 (2010).

[10] M. Berasaluce-Gonzalez, P. G. Camara, F. Marchesano, D. Regalado, and A. M. Uranga, J. High Energy Phys. 09 (2012) 059.

[11] F. Marchesano, D. Regalado, and L. Vazquez-Mercado, J. High Energy Phys. 09 (2013) 028.

[12] H. Abe, T. Kobayashi, H. Ohki, K. Sumita, and Y. Tatsuta, J. High Energy Phys. 06 (2014) 017.

[13] T. Higaki, N. Kitazawa, T. Kobayashi, and K. j. Takahashi, Phys. Rev. D 72, 086003 (2005).

[14] T. Kobayashi and S. Nagamoto, Phys. Rev. D 96, 096011 (2017).

[15] D. Cremades, L. E. Ibanez, and F. Marchesano, J. High Energy Phys. 05 (2004) 079.

[16] J. Lauer, J. Mas, and H. P. Nilles, Phys. Lett. B 226, 251 (1989); Nucl. Phys. B351, 353 (1991).

[17] W. Lerche, D. Lust, and N. P. Warner, Phys. Lett. B 231, 417 (1989).
[18] S. Ferrara, D. Lust, and S. Theisen, Phys. Lett. B 233, 147 (1989).

[19] H. Abe, K. S. Choi, T. Kobayashi, and H. Ohki, J. High Energy Phys. 06 (2009) 080.

[20] H. Abe, T. Kobayashi, and H. Ohki, J. High Energy Phys. 09 (2008) 043.

[21] T. Kobayashi and N. Ohtsubo, Phys. Lett. B 245, 441 (1990); Int. J. Mod. Phys. A 09, 87 (1994).

[22] T. H. Abe, Y. Fujimoto, T. Kobayashi, T. Miura, K. Nishiwaki, and M. Sakamoto, J. High Energy Phys. 01 (2014) 065; Nucl. Phys. B890, 442 (2014).

[23] Y. Hamada and T. Kobayashi, Prog. Theor. Phys. 128, 903 (2012).

[24] S. Ferrara, D. Lust, A. D. Shapere, and S. Theisen, Phys. Lett. B 225, 363 (1989).

[25] S. Ferrara, N. Magnoli, T. R. Taylor, and G. Veneziano, Phys. Lett. B 245, 409 (1990).

[26] M. Cvetic, A. Font, L. E. Ibanez, D. Lust, and F. Quevedo, Nucl. Phys. B361, 194 (1991).

[27] J. P. Derendinger, S. Ferrara, C. Kounnas, and F. Zwirner, Nucl. Phys. B372, 145 (1992).

[28] L. E. Ibanez and D. Lust, Nucl. Phys. B382, 305 (1992).

[29] L. E. Ibanez, D. Lust, and G. G. Ross, Phys. Lett. B 272, 251 (1991).

[30] H. Kawabe, T. Kobayashi, and N. Ohtsubo, Nucl. Phys. B434, 210 (1995).

[31] T. Kobayashi, S. Nagamoto, and S. Uemura, Prog. Theor. Exp. Phys. 2017, 023B02 (2017).

[32] T. Araki, T. Kobayashi, J. Kubo, S. Ramos-Sanchez, M. Ratz, and P. K. S. Vaudrevange, Nucl. Phys. B805, 124 (2008).

[33] J. Talbert, arXiv:1804.04237.

[34] F. Feruglio, arXiv:1706.08749.

[35] T. Kobayashi, K. Tanaka, and T. H. Tatsuishi, arXiv: 1803.10391. 